\title{
Polarization rotator based on augmented low-index-guiding waveguide on silicon nitride/silicon-on-insulator platform
}

\author{
X. Sun, ${ }^{1, *}$ M. Z. Alam, ${ }^{2}$ J. S. Aitchison,,${ }^{1}$ and M. Mojahedi ${ }^{1}$ \\ ${ }^{1}$ Department of Electrical \& Computer Engineering, University of Toronto, 10 King's College Road, Toronto, Ontario M5S3G4, Canada \\ ${ }^{2}$ Department of Applied Physics and Materials Science, California Institute of Technology, Pasadena, California 91106, USA \\ ${ }^{*}$ Corresponding author: xiaoxiao.sun@utoronto.ca
}

Received 29 March 2016; revised 12 June 2016; accepted 16 June 2016; posted 17 June 2016 (Doc. ID 262119); published 8 July 2016

Using a newly proposed augmented low-index-guiding scheme with silicon nitride/silicon dual-core waveguide, we have designed, fabricated, and characterized a transverse electric (TE) to transverse magnetic (TM) and TM-to-TE compact polarization rotator. The polarization rotation is realized in an asymmetric directional coupler. The measured peak conversion efficiencies for the TE-to-TM and TM-to-TE rotations are approximately $97 \%$. The measured polarization extinction ratio for the TE-to-TM rotation is greater than $20 \mathrm{~dB}$ over $50-\mathrm{nm}$ bandwidth, while for the TM-to-TE rotation it is greater than $15 \mathrm{~dB}$ over the C-band. (๑) 2016 Optical Society of America

OCIS codes: (130.2790) Guided waves; (130.3120) Integrated optics devices; (130.5440) Polarization-selective devices; (230.7370) Waveguides.

http://dx.doi.org/10.1364/OL.41.003229

Control polarization in photonic integrated circuits (PICs) and in particular for platforms having a high-index contrast is important $[1,2]$. The propagation properties of the transverse electric (TE) to transverse magnetic (TM) modes in a highcontrast waveguide are quite different; hence, polarizationsensitive photonic devices for such platforms must be carefully designed. When designing a PIC, one device often needed is the polarization rotator (PR). Such a device will allow the user to rotate one polarization state to the other one, for example, TE-to-TM mode.

Several techniques can realize a PR. For example, the authors in [3-5] have used an asymmetric axis-rotated section to achieve the required polarization rotation. These approaches are based on complicated fabrication steps such as off-axis double core structure [3], polycrystalline silicon overlay [4], or precise partial etching [5]. Another technique is adiabatic mode evolution [6-8]. However, this approach requires long device lengths and high aspect ratios, and in some cases several polarization beam splitters to enhance the polarization extinction ratio (PER) and performance [8]. The PR which is based on the plasmonic waveguide can reduce the device length; however, it requires extra fabrication steps for metal deposition and has a high insertion loss [9]. Last, when designing PRs, it seems an asymmetrical directional coupler is the preferred technique due to its relatively simple fabrication and broadband response [10-12]. However, to break the symmetry-to couple out and rotate one of the orthogonal polarizations-air cladding (instead of conventional upper silica cladding) is needed. For PICs with thick silica cladding, other methods such as partial etching of the waveguide have been used [13-15], increasing the number of etch steps and the possibility of misalignment among different layers.

In this Letter, we report the design and experimental demonstration of a PR based on a new platform - the augmented low-index-guiding (ALIG) waveguide. Compared to other PRs using polarization coupling between two adjacent waveguides, the proposed device performs well even when silica cladding is included. Taking advantage of the recent mature techniques in using silicon nitride in PIC design and fabrication [16,17], the device fabrication is straightforward with the silicon nitride/ silicon dual-core waveguide structure. In addition, it does not require alignment of different layers.

The ALIG waveguide was first proposed in [18]. The ALIG waveguide consists of a thinned silicon layer and a silicon nitride layer working together as a core. The schematic of the ALIG waveguide is shown in Fig. 1(a). The thickness of the silicon layer is $t_{1}$, and the thickness of the silicon nitride layer is $t_{2}$. In such a structure, $t_{1}$ is much thinner than that of a standard silicon-on-insulator (SOI) waveguide.

An interesting outcome of the above is that for the TM mode, with properly chosen dimensions, the light intensity is strongest in the low-index region (i.e., silicon nitride). The
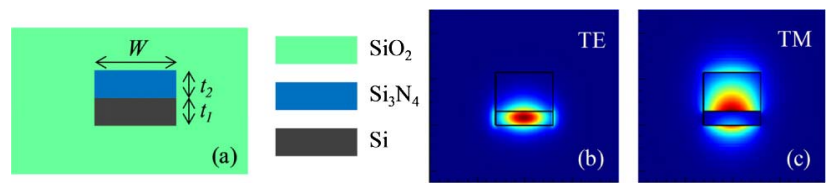

Fig. 1. (a) Schematic structure (cross section) of the ALIG waveguide. (b) Intensity for the TE mode. (c) Intensity for the TM mode. $t_{1}=120 \mathrm{~nm}, t_{2}=340 \mathrm{~nm}, W=600 \mathrm{~nm}$. 
analysis of the relation between dimensions and power distribution for the TM mode shows that for $t_{1}=90-120 \mathrm{~nm}$ and $t_{2} \geq 300 \mathrm{~nm}$ most of power can be concentrated in the silicon nitride layer [19]. In that case, the TM mode is mainly guided by the silicon nitride layer. On the other hand, the TE mode of the structure remains undisturbed and guided by the highindex silicon layer. The mode profiles of a typical single mode ALIG waveguide are simulated using Lumerical MODE Solutions, and the intensities are shown in Figs. 1(b) and 1(c), with $t_{1}=120 \mathrm{~nm}, t_{2}=340 \mathrm{~nm}$, and $W=600 \mathrm{~nm}$. The fact that TE and TM modes reside in two different layers provides the designer with more flexibility for on-chip polarization control. A polarization beam splitter based on ALIG waveguide has been reported in [20].

Because the ALIG waveguide has intrinsic asymmetry in the vertical direction, coupling out and rotating one of the two orthogonal polarizations is possible using a simple directional coupler. Figure 2(a) shows a three dimensional schematic of the proposed PR using a directional coupler. The device consists of an input waveguide, followed by an asymmetric coupling section (formed by two adjacent branches with different widths), and output waveguides. To be consistent with the conventional PIC design rules, the whole device is covered with silica upper cladding. Figure 2(b) shows the cross section of the coupling region where the widths of the two adjacent waveguides are $W_{1}$ and $W_{2}$, respectively. The center-to-center distance between the two waveguides is $D$.

To design the coupling section of the PR, we have calculated the effective index $\left(n_{\text {eff }}\right)$ of a single ALIG waveguide, at the wavelength of $1.55 \mu \mathrm{m}$, for different waveguide widths from 300 to $700 \mathrm{~nm}$ with fixed thickness $t_{1}=120 \mathrm{~nm}$ and $t_{2}=340 \mathrm{~nm}$. In our simulations the refractive indices for silicon, silicon nitride, and silica are 3.476, 2, and 1.444, respectively. These results are shown in Fig. 3. From the figure, clearly the effective index of the fundamental TE mode (TE0) is larger than the fundamental TM mode (TM0) for the same width. To meet the phase matching condition of the two orthogonal polarizations, the waveguide supporting the TM mode should be wider than the one supporting the TE mode. We have chosen $W_{1}=600 \mathrm{~nm}$ for the wider waveguide and $W_{2}=345 \mathrm{~nm}$ for the narrower one. We note that for these dimensions the higher order TE mode (TE1) is leaky and can be ignored. Thus under these circumstances the ALIG waveguide is single mode, which only guides the fundamental TE or TM modes.

When the two waveguides are put close enough, coupled modes of hybridized polarization form. One polarization will be converted to the other polarization in the asymmetric directional coupler. In designing the coupler section we note that
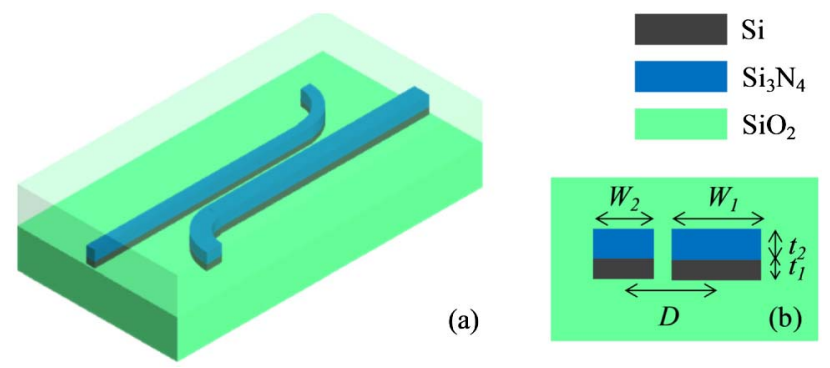

Fig. 2. (a) Three-dimensional schematic of the PR. (b) Cross section of the coupler section.

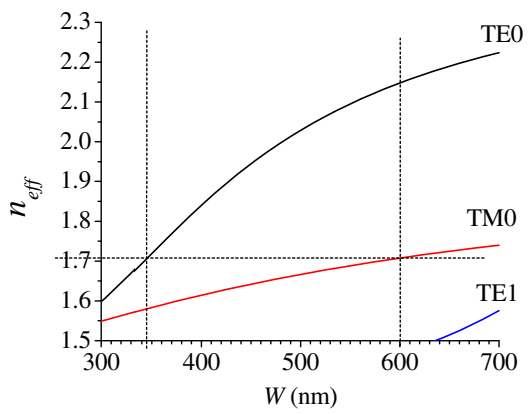

Fig. 3. Effective indices of the modes in the ALIG waveguide with silica cladding for different widths with fixed $t_{1}=120 \mathrm{~nm}$, $t_{2}=340 \mathrm{~nm}$. The working wavelength is $1.55 \mu \mathrm{m}$.

while a smaller gap can reduce the coupling length, considering fabrication tolerances and ease of fabrication, a larger gap is preferred. In our design we have chosen $D$ to be $700 \mathrm{~nm}$. This dimension is large enough for either electron beam lithography or deep UV lithography.

The proposed PR can work for either TE-to-TM or TM-toTE conversion, when the input light is launched from different ports. As an example, consider the TE-to-TM conversion depicted schematically in Fig. 4(a), where the length of the coupling section is $L$. When the input TE0 light is launched in the narrower waveguide $\left(W_{2}=345 \mathrm{~nm}\right)$, it is coupled to the TM0 of the wider waveguide $\left(W_{1}=600 \mathrm{~nm}\right)$, through the coupling section. The uncoupled TE0 light will continue on the narrow waveguide to the residue port where it will be discarded.

The case of TM-to-TE conversion is depicted in Fig. 4(b), where the TM mode is launched from the wider $\left(W_{1}=600 \mathrm{~nm}\right)$ waveguide. In the coupling region, the TM mode couples to the TE mode of the narrower waveguide $\left(W_{2}=345 \mathrm{~nm}\right)$, and the remaining uncoupled TM light will continue to the residue port where it will be discarded.

We have calculated the $n_{\text {eff }}$ for the hybridized supermodes and estimated the corresponding coupling length $(L)$ is about $40 \mu \mathrm{m}$. Considering the possible coupling in the bends, final length $L$ is determined using finite-difference time-domain (FDTD) method; the optimized length is $L=35 \mu \mathrm{m}$. To transition smoothly and guide the unwanted residue light,

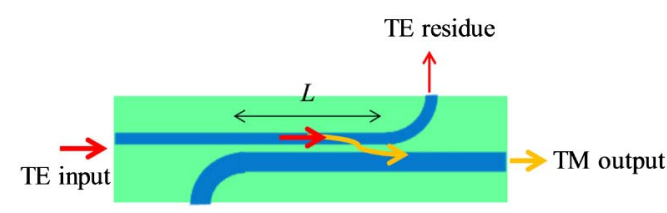

(a)

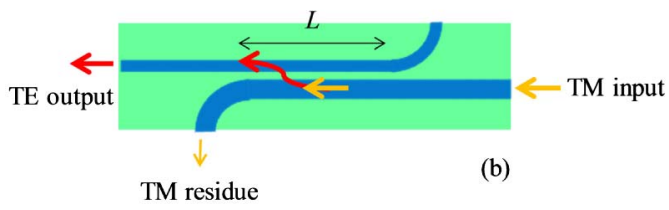

Fig. 4. (a) Top view of the PR and the schematics for TE-to-TM mode conversion. (b) Top view of the PR and the schematics for TM-to-TE mode conversion. 
bends are used to route the light. According to simulation, the radiation loss is negligible for both modes when the bend radius is $15 \mu \mathrm{m}$.

For the directional coupler with a smooth transition, the device has a very low reflection loss (less than $-40 \mathrm{~dB}$ ). Also, we consider the propagation loss is very low for the $35-\mu \mathrm{m}$-long device only. The input power just before the rotator can be treated as the sum of output powers in the two output waveguides immediate after the rotator. Here we focus on two important parameters for a PR: conversion efficiencies and PERs. The conversion efficiency $(\eta)$, for example for the TE-to-TM rotation, is defined as the power of the TM mode in the TM output port to the total power in both TM output and TE residue ports, where the port names are defined in Fig. 4(a):

$$
\eta_{\text {TE-TM }}=\frac{{ }^{\mathrm{TM}} P_{\text {TMoutput }}}{{ }_{\text {total }} P_{\text {TMoutput }}+{ }^{\text {total }} P_{\text {TEresidue }}} .
$$

On the other hand, the PER for the TE-to-TM conversion is defined as the power of the TM mode to the power of TE mode in the TM output port:

$$
\mathrm{PER}_{\mathrm{TE}-\mathrm{TM}}=10 \log 10\left(\frac{{ }^{\mathrm{TM}} P_{\text {TMoutput }}}{{ }^{\mathrm{TE}} P_{\text {TMoutput }}}\right) .
$$

Similar expressions also apply to the TM-to-TE conversion.

We have used the FDTD method to calculate the aforementioned parameters for our design. Also, to extract the different polarization components from the output ports, mode expansion monitors are used to analyze the modal overlap and calculate the transmissions for different modes.

The conversion efficiency measures the fraction of power properly converted; in other words, the unconverted part can be treated as an insertion loss for the PR. Figure 5 shows the results over $50 \mathrm{~nm}$ of bandwidth for both TE-to-TM and TM-to-TE conversions. From the figure it is clear that conversion efficiencies for both modes are greater than $67 \%$, so the insertion loss is less than $1.7 \mathrm{~dB}$ over the $50-\mathrm{nm}$ bandwidth. At the center bandwidth, from 1550 to $1560 \mathrm{~nm}$, the conversion efficiency is $97 \%$ and the corresponding insertion loss is less than $0.2 \mathrm{~dB}$.

The PER measures polarization purity at the output port. Figure 6 shows the calculated PER for both TE-to-TM and TM-to-TE conversions. As the figure indicates, greater than 20-dB PER is achieved over 50-nm bandwidth for the TEto-TM conversion. On the other hand, the PER for the TM-to-TE conversion is greater than $15 \mathrm{~dB}$ over the C-band (1530 to $1565 \mathrm{~nm})$. The lower PER for the TM-to-TE rotation

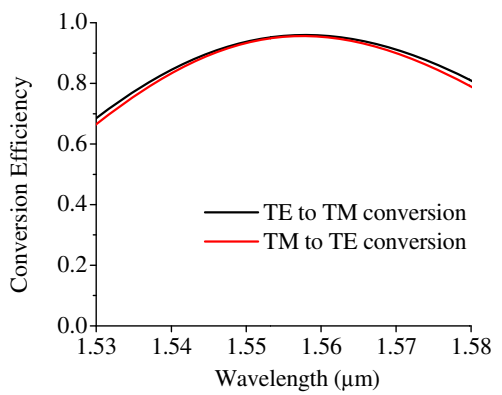

Fig. 5. Simulated conversion efficiency for both TE-to-TM and TM-to-TE conversions.

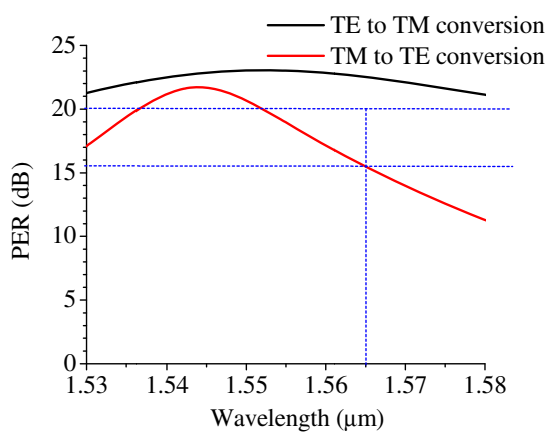

Fig. 6. Calculated PER for both TE-to-TM and TM-to-TE conversions.

is expected, because as noted in Fig. 3 the difference of effective indices for the TM0 modes in the wider and the narrower waveguides are not as large as the difference for the TE0 modes in these two waveguides. Some portion of the power for the TM0 mode in wider waveguide still likely couples to the TM0 mode in the narrower waveguide although the large portion of the power is converted to TE0 mode.

We have used silicon compatible processes to fabricate the ALIG waveguides. The waveguides were fabricated using a standard silicon-on-insulator (SOI) wafer with a 220 -nm device layer on top of a $2-\mu \mathrm{m}$ buried oxide layer. The SOI wafer was placed in the oxidation furnace, and a wet oxidation process was used to form a layer of oxide film which consumes the top 100$\mathrm{nm}$ silicon layer. Next, the oxide layer was removed using buffered oxide etching, thinning the device layer to only $120 \mathrm{~nm}$. Later, a 340-nm layer of silicon nitride was deposited on the wafer using low-pressure chemical vapor deposition. The ALIG wafer was then cleaved into dies. Samples were coated with resist, and patterns were defined using electron beam lithography. The patterns were then transferred using the reactive ion etching process with mixed gas of $\mathrm{SF}_{6}$ and $\mathrm{C}_{4} \mathrm{~F}_{8}$. For the fully etched waveguides, both silicon nitride and silicon layers were etched during the same run, and the etching recipe was optimized to guarantee straight sidewalls. In the final step of fabrication, a thick layer of flowable oxide was spin-coated over the sample and heated to form the top silica cladding. A scanning electron microscope (SEM) picture of a fabricated PR, before depositing the upper flowable oxide cladding, is shown in Figs. 7(a) and 7(b). The input and output waveguides and the residual ports are all extended to the edge of the die for free space coupling and measurements. Several straight waveguides were also fabricated on the same die to measure
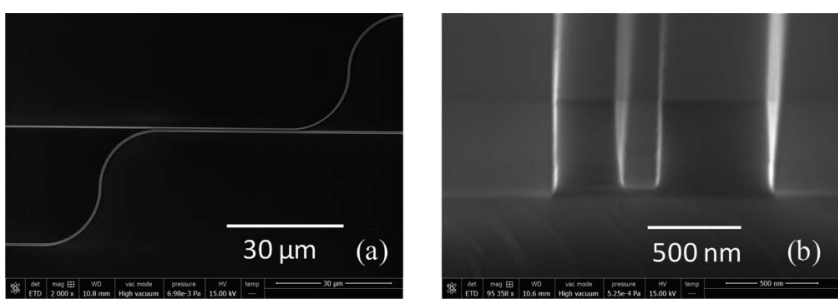

Fig. 7. SEM images of the fabricated polarization convertor before deposition of upper cladding. (a) Top view. (b) Cross section of the asymmetric directional coupler part. 


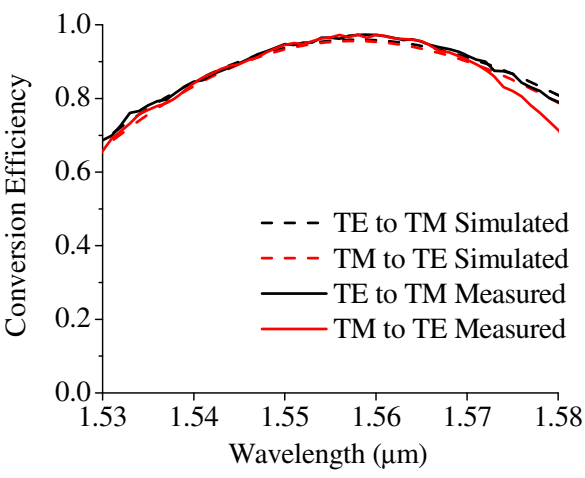

Fig. 8. Measured conversion efficiency for both TE-to-TM and TM-to-TE conversions.

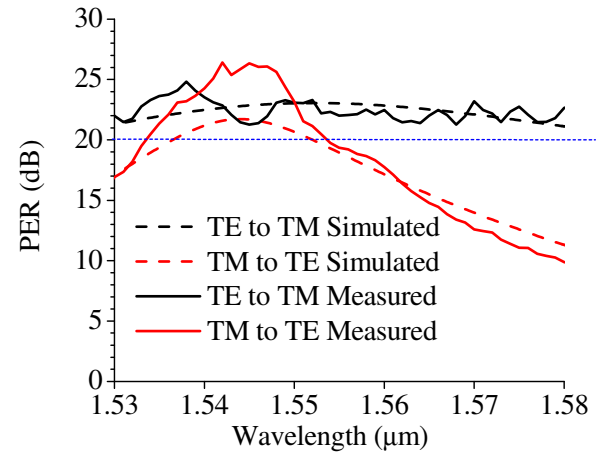

Fig. 9. Measured PER for both TE-to-TM and TM-to-TE conversions.

and calibrate-out the propagation losses associated with the TM and TE polarizations.

To characterize the fabricated devices, a superluminescent diode source together with erbium-doped fiber amplifiers were used as a broadband source. The light was collimated to a free space beam. Two polarization beam splitter cubes-one before and another after the sample-were used to control the input polarizations into the sample, and to detect and separate the polarization states of the light at the sample's output. A pair of $x 40$ objective lenses was used to couple light into the sample and collect the output. Finally, the light from the sample's output port was routed to an optical spectrum analyzer in order to detect and characterize its spectrum.

Figure 8 shows the measured and calculated (the same as Fig. 5) conversion efficiencies for both TE-to-TM and TMto-TE rotations. As the figure indicates, for both the TE-toTM and TM-to-TE rotations, the measured peak conversion efficiency is approximately $97 \%$, which corresponds to only a $0.2-\mathrm{dB}$ insertion loss; with an overall insertion loss of better than $1.7 \mathrm{~dB}$ over the $50 \mathrm{~nm}$ bandwidth.

The measured and calculated (from Fig. 6) PERs for both TE-to-TM and TM-to-TE conversions are shown in Fig. 9. It is observed that PER for TE-to-TM conversion is greater than $20 \mathrm{~dB}$ over $50-\mathrm{nm}$ bandwidth. On the other hand, the PER for the TM-to-TE conversion is greater than $15 \mathrm{~dB}$ over the C-band (1530 to $1565 \mathrm{~nm})$. We found a slight discrepancy for the PER of the TM-to-TE conversion, possibly because of slight changes in dimension due to the fabrication process.

In summary, we have designed and experimentally demonstrated a compact PR based on the novel ALIG waveguide where the guiding layers consist of silicon nitride/silicon layers. Using an asymmetric directional coupler section, the device works for both TE-to-TM and TM-to-TE rotations when the input light is launched from different ports. It has an overall insertion loss of better than $1.7 \mathrm{~dB}$ over the $50-\mathrm{nm}$ bandwidth, with PER for the TE-to-TM rotation greater than $20 \mathrm{~dB}$ over 50-nm bandwidth and for the TM-to-TE rotation greater than $15 \mathrm{~dB}$ over the C-band. The device is simple to fabricate and works well with silica over-cladding, which is required in photonics integrated circuits packaging.

Funding. Natural Sciences and Engineering Research Council of Canada (NSERC).

\section{REFERENCES}

1. D. Dai, J. Bauters, and J. E. Bowers, Light 1, e1 (2012).

2. H. Fukuda, K. Yamada, T. Tsuchizawa, T. Watanabe, H. Shinojima, and S. Itabashi, Opt. Express 16, 4872 (2008).

3. H. Fukuda, K. Yamada, T. Tsuchizawa, T. Watanabe, H. Shinojima, and S. Itabashi, Opt. Express 16, 2628 (2008).

4. D. Vermeulen, S. Selvaraja, P. Verheyen, P. Absil, W. Bogaerts, D. V. Thourhout, and G. Roelkens, IEEE Photon. Technol. Lett. 24, 482 (2012).

5. Z. Wang and D. Dai, J. Opt. Soc. Am. B 25, 747 (2008).

6. M. R. Watts and H. A. Haus, Opt. Lett. 30, 138 (2005).

7. J. Zhang, M. Yu, G. Lo, and D. Kwong, IEEE J. Sel. Top. Quantum Electron. 16, 53 (2010)

8. L. Chen, C. R. Doerr, and Y. Chen, Opt. Lett. 36, 469 (2011).

9. J. Niklas Caspers, J. Stewart Aitchison, and M. Mojahedi, Opt. Lett. 38, 4054 (2013).

10. D. Dai and J. E. Bowers, Opt. Express 19, 10940 (2011).

11. L. Liu, Y. Ding, K. Yvind, and J. M. Hvam, Opt. Lett. 36, 1059 (2011).

12. Y. Ding, L. Liu, C. Peucheret, and H. Ou, Opt. Express 20, 20021 (2012).

13. H. Guan, A. Novack, M. Streshinsky, R. Shi, Q. Fang, A. E.-J. Lim, G.-Q. Lo, T. Baehr-Jones, and M. Hochberg, Opt. Express 22, $2489(2014)$

14. Y. Xiong, D. Xu, J. H. Schmid, P. Cheben, S. Janz, and W. N. Ye, Opt. Express 22, 17458 (2014).

15. W. D. Sacher, T. Barwicz, B. J. F. Taylor, and J. K. S. Poon, Opt. Express 22, 3777 (2014).

16. W. D. Sacher, Y. Huang, G.-Q. Lo, and J. K. S. Poon, J. Lightwave Technol. 33, 901 (2015).

17. K. Shang, S. Pathak, B. Guan, G. Liu, and S. J. B. Yoo, Opt. Express 23, 21334 (2015).

18. M. Z. Alam, X. Sun, M. Mojahedi, and J. S. Aitchison, in CLEO, OSA Technical Digest (Optical Society of America, 2015), paper FW1D.2.

19. X. Sun, M. Z. Alam, J. S. Aitchison, and M. Mojahedi, in CLEO, OSA Technical Digest (Optical Society of America, 2015), paper JTu5A.52.

20. X. Sun, M. Z. Alam, J. S. Aitchison, and M. Mojahedi, Opt. Lett. 41, 163 (2016). 